Hippocampus

\title{
Lesions of the mammillothalamic tract and anterior thalamic nuclei: Response to Vann and Nelson (2018)
}

\begin{tabular}{|r|l|}
\hline Journal: & Hippocampus \\
\hline Manuscript ID & HIPO-18-041.R2 \\
\hline Wiley - Manuscript type: & Letter to the Editor \\
\hline Keywords: & $\begin{array}{l}\text { Diencephalic amnesia, Anterior thalamic nuclei, Mammillothalamic tract, } \\
\text { Lesions, zif268 }\end{array}$ \\
\hline \multicolumn{2}{|c}{} \\
\hline
\end{tabular}

\section{SCHOLARONE \\ Manuscripts}


Title: Lesions of the mammillothalamic tract and anterior thalamic nuclei: Response to Vann and Nelson (2018)

John C. Dalrymple-Alford (1) and Brook A. L. Perry (2)

(1) Department of Psychology, New Zealand Brain Research Institute

University of Canterbury and Brain Research New Zealand

Christchurch 8041

New Zealand

Email: jc.dalrymple.alford@gmail.com

(2) Department of Experimental Psychology

University of Oxford

Tinsley Building

Mansfield Road

Oxford. OX1 3SR

United Kingdom

Email: brook.perry@psy.ox.ac.uk 
Dear Editor,

We thank Vann and Nelson (2018) for providing the opportunity for further discussion on our paper, "Anterior thalamic nuclei lesions have a greater impact than mammillothalamic tract lesions on the extended hippocampal system", by Perry, Mercer, Barnett, Lee and DalrympleAlford (2018). We contend that our findings addressed important unexplored questions concerning the effects of anterior thalamic nuclei (ATN) lesions and mammillothalamic tract (MTT) lesions.

Their key point was to suggest that we have replicated previous findings on ATN and MTT lesion effects in the water-maze and radial arm maze. However, relatively few studies have examined discrete MTT lesions and there have been inconsistent findings. Our withinstudy comparison of ATN and MTT lesions obviates procedural differences between independent studies that may underpin their different findings. For example, one study examined the effects of MTT lesions on two spatial memory tasks in the water-maze and reported the opposite of what might be expected: no impairment in working memory but an impairment, albeit mild, in reference memory (Winter, Wagner, McMillin, \& Wallace, 2011). A second example, by contrast, used a standard T-maze alternation procedure and MTT lesions produced a profound working memory deficit that persisted across training (Frizzati et al., 2016), despite previous testing on several other visuospatial memory tasks (Nelson \& Vann, 2014). In other T-maze alternation reports, rats with MTT lesion effects have shown rapidly improving performance across repeat testing, at least for standard test procedures, whereas ATN lesions generally produce more persistent impairments (Aggleton and Nelson, 2015; Aggleton, Poirier, Aggleton, Vann, \& Pearce, 2009; Loukavenko, Ottley, Moran, Wolff, \& Dalrymple-Alford, 2007; Loukavenko, Wolff, Poirier, \& Dalrymple-Alford, 2016; Ulrich, Aitken, Abraham, Dalrymple-Alford, \& McNaughton, 2014; Vann \& Aggleton, 2003; Vann, 2013; Warburton, Morgan, Baird, Muir \& Aggleton, 1999). With respect to Vann and 
Nelson's (2018) reference to mammillary body (MB) lesion studies, Vann and colleagues have elsewhere pointed out that MTT lesions provide a direct test of the functional significance of MB inputs to the ATN (Vann \& Aggleton, 2003; Vann, 2010; Dillingham, Frizzati, Nelson, \& Vann, 2015) whereas MB lesion studies are not optimal for this purpose due to the complexity of this region and frequent confounds with non-specific injury (Gonzalo-Ruiz, Alonso, Sanz, \& Llinas, 1992; Kirk, 1998).

Their second focus was that, in the abstract, we stated, "it is assumed that their (the MTT and ATN) influence on memory is functionally equivalent." Our sentence began by linking this comment to human anterograde amnesia, which provides the impetus for animal studies on diencephalic support of memory function. In the clinical context it is generally assumed that the core features of different examples of diencephalic amnesia are fundamentally the same and reflect disruption to the MB-ATN axis. As mentioned in our paper (Perry et al., 2018), the evidence from diencephalic amnesia as a consequence of stroke or infarct most consistently implicates MTT injury as causal for severe amnesia (e.g. Carlesimo, Lombardi, \& Caltagirone, 2011; but see Duprez, Serieh, \& Raftopoulos, 2005), whereas the strongest direct evidence to implicate the ATN comes from alcoholic Korsakoff patients (Kopelman, 2015). It is suggested that a greater range of impairments in the latter patient group may stem primarily from additional dysfunction, especially to the prefrontal cortex and the mediodorsal nucleus (Kopelman, 2015). Vann and Nelson (2018) also mention other extracts from our paper but incorrectly assert that we implied that all ATN function might be entirely explained by MTT and brainstem injury. Instead, our question was the degree of similarity, and severity, of ATN and MTT lesion effects in terms of memory tasks and related neurobiology. These issues are relevant given the limited and uncertain evidence from experimental MTT studies and the weight given to MTT lesions in the clinical 
literature. Clarity on the relative effects of ATN and MTT injury is critical to determine their potential impact for human cases of amnesia (Danet et al., 2015).

Another important issue is the impact of ATN and MTT lesions on immediate early gene (IEG) activity in limbic cortex. Vann and Nelson (2018) want us to clarify that our failure to observe MTT lesion effects for zif268 expression in the deep layers of the granular b retrosplenial cortex $(\mathrm{Rgb})$, and in the dysgranular retrosplenial cortex, differed from their recent report on MTT lesion effects (Frizzati et al., 2016). That study looked at zif268 in brain sections from the same rats previously examined for a c-Fos study (Vann and Albasser, 2009). However, the zif268 data in Figure 4B of Frizzati et al. (2016) did not align with their statements concerning a much larger effect of MTT lesions on c-Fos, so we avoided comment due to this uncertainty. The more important question, however, is the direct within-study comparison of MTT and ATN lesions on IEG measures in the limbic system, because previous studies have produced variable findings that depend, for example, on the time since lesion surgery and the IEG marker in question (Dumont, Amin, Poirier, Albasser, \& Aggleton, 2012; Dupire et al., 2013; Jenkins, Dias, Amin, \& Aggleton, 2002a; Jenkins, Dias, Amin, Brown, \& Aggleton, 2002b; Jenkins, Amin, Pearce, Brown, \& Aggleton, 2004; Loukavenko, Wolff, Poirier, \& Dalrymple-Alford, 2016; Poirier et al., 2008; Poirier \& Aggleton, 2009). Our zif268 findings shed new light on the proposal that "The qualitative and quantitative equivalence of anterior thalamic and mammillothalamic tract lesion effects on the pattern of [IEG; our insertion] dysfunction observed in distal regions raises the possibility that these anterior thalamic lesion effects are principally driven by the loss of their mammillary body inputs" (Dillingham et al., 2015, p.115; see also, Vann, 2010, p.2323, and Frizzati et al., 2016, p.47). We found that both ATN and MTT lesions substantially and equally reduced zif 268 expression in the superficial layers of the rostral Rgb; in this regard, our results support the proposal of Dillingham et al. (2015). There was, however, a 
significantly greater reduction of zif268 expression in the caudal Rgb, and in hippocampal CA1, after ATN lesions than MTT lesions; and only ATN lesions reduced zif268 expression in the deep layers of Rgb, in superficial layers of the Rga and in dysgranular retrosplenial cortex. We think it unlikely that these differences may have arisen from behavioural variation between our groups just prior to euthanasia. Our procedure permitted a maximum of 8 arm visits (four before and after maze rotation) for all rats on each of three massed trials, we ensured similar food rewards regardless of performance and, critically, the last day produced a mean difference of less than one error per trial between the sham group and both lesion groups (group effect, last day, $\mathrm{F}(2,41)=2.58, \mathrm{p}<0.1$, with no pairwise differences; our Figure 5c, day 3).

In addition, Vann and Nelson (2018) ask whether our effects were due to a less stringent criterion for MTT lesions. We used luxol blue myelin stain to confirm the loss of MTT fibres, directly, whereas all other studies used a Nissl stain (cresyl violet) for the assessment of the MTT lesion; one study added retrograde tracers in the ATN in one rat to suggest that lateral MB efferents are unaffected despite a total MTT lesion assessed by Nissl stain (Vann \& Albasser, 2009). We should have explicitly stated that five of our rats had $100 \%$ bilateral MTT lesions after assessing the luxol blue stain, while four other rats with acceptable lesions had a bilateral minima of $89 \%, 96 \%, 99 \%$ and $99 \%$ injury; five other rats were discarded that had primarily unilateral lesions. We found that the included MTT lesions produced a reduction in relative neuronal cell counts in all three subregions of the MB; this effect was greater than that produced by our far smaller percentage-size lesions made in the ATN (Perry et al., 2018, Figure 6). These changes in the MB are expected from anatomical studies of the MTT (Cruce, 1975; Guillery, 1957; Seki \& Zyo, 1984), but is a potential difference from Vann and colleagues' MTT lesions, which may leave efferents of the lateral MB unaffected (Vann \& Albasser, 2009). Variation in the anatomical placement of the MTT 
lesion may be one reason for these differences, and they highlight the need for more evidence on the behavioural and functional impact of MTT lesions.

The final point made by Vann and Nelson (2018) was that we did not give an accurate representation of lesion effects on the geometric task in the water-maze, in which rats are discouraged from using allocentric cues and must use the shape of the walled environment to identify the position of a hidden platform. They propose that differences across lesions reflect a distinction between head direction and non-head direction regions of the MB and the ATN. This is a possibility, but does not reconcile the different effects of MTT, lateral MB and ATN lesions on this geometric task. Lateral MB lesions, which would remove head direction information from this region to the anterodorsal ATN, produced a transient acquisition deficit in this task (Vann, 2011). By contrast, neither fornix lesions, total ATN lesions (which included the anterodorsal region) nor MTT lesions (which may have excluded lateral MB efferents to the anterodorsal region) have produced a significant acquisition deficit on this task (Aggleton, Poirier, Aggleton, Vann and Pearce, 2009; Vann, 2013). These differences may in part reflect the faster acquisition by sham controls in the lateral MB lesion study compared to the sham groups in other studies. Moreover, rats with lateral MB lesions, fornix lesions, or MTT lesions showed normal performance on the subsequent probe trial when the platform was removed, whereas a profound deficit on the probe trial was produced by total ATN lesions irrespective of active or passive learning experience during acquisition (Aggleton et al., 2009; Dumont, Wright, Pearce and Aggleton, 2014). Whether head direction or other processes are important in geometric learning is therefore uncertain. It is possible that an interaction of head-direction information between limbic system cortex and the ATN remains an important factor on the probe trial in the geometric task. In addition, the integration of head-direction information and theta processing in neurons in the medial anteroventral nucleus of the ATN suggests that a separation between head direction and theta 
at the level of the ATN over-simplifies the influence of this region on memory processes in upstream limbic structures (Tsanov et al., 2011).

We appreciate Vann and Nelson's (2018) interest in our study and are respectful of the valuable contributions that they and other co-authors have made. Their commentary claimed that we have generated a "straw-man" argument. This suggests that we have attributed something that they do not claim, equivalent impairments after ATN and MTT lesions. Our paper did not, however, attribute a specific model to them in terms of ATN and MTT lesions. As reiterated above, we addressed genuine questions driven by the clinical literature, especially, as well as animal literature, concerning the relative impact of MTT and ATN injury or dysfunction. While these authors have suggested that ATN lesions and MTT lesions have equivalent effects on IEG changes in the retrosplenial cortex, and our data contribute to this issue, this need not be relevant to all the effects of the two lesions. In summary, our original paper illustrates the value of a direct comparison of ATN and MTT lesions, much like other comparisons such as ATN and fornix lesions. The role played by the MTT and the ATN, in particular, in concert with other subcortical and cortical structures, provides a fruitful way to understand the neural basis of memory and to explore potentials for recovery of function when memory is impaired (Dalrymple-Alford et al., 2015; Aggleton, Pralus, Nelson, \& Hornberger, 2016).

Yours sincerely, John C. Dalrymple-Alford and Brook A. L. Perry 06 May 2018 


\section{References}

Aggleton, J. P., \& Nelson, A. J. (2015). Why do lesions in the rodent anterior thalamic nuclei cause such severe spatial deficits? Neuroscience \& Biobehavioral Reviews, 54, 131-144.

Aggleton, J. P., Pralus, A., Nelson, A. J., \& Hornberger, M. (2016). Thalamic pathology and memory loss in early Alzheimer's disease: moving the focus from the medial temporal lobe to Papez circuit. Brain, 139(7), 1877-1890.

Aggleton, J. P., Poirier, G. L., Aggleton, H. S., Vann, S. D., \& Pearce, J. M. (2009). Lesions of the fornix and anterior thalamic nuclei dissociate different aspects of hippocampaldependent spatial learning: Implications for the neural basis of scene learning. Behavioral Neuroscience, 123(3), 504.

Carlesimo, G. A., Lombardi, M. G., \& Caltagirone, C. (2011). Vascular thalamic amnesia: A re appraisal. Neuropsychologia, 49, 777-789.

Cruce, J. A. (1975). An autoradiographic study of the projections of the mammillothalamic tract in the rat. Brain Research, 85(2), 211-219.

Dalrymple-Alford, J. C., Harland, B., Loukavenko, E. A., Perry, B., Mercer, S., Collings, D. A., ... \& Wolff, M. (2015). Anterior thalamic nuclei lesions and recovery of function: relevance to cognitive thalamus. Neuroscience \& Biobehavioral Reviews, 54, 145-160. Danet, L., Barbeau, E. J., Eustache, P., Planton, M., Raposo, N., Sibon, I., . . Pariente, J. (2015). Thalamic amnesia after infarct: The role of the mammillothalamic tract and mediodorsal nucleus. Neurology, 85(24), 2107-2115.

Dillingham, C. M., Frizzati, A., Nelson, A. J., \& Vann, S. D. (2015). How do mammillary body inputs contribute to anterior thalamic function? Neuroscience \& Biobehavioral Reviews, 54, 108-119. 
Dumont, J. R., Amin, E., Poirier, G. L., Albasser, M. M., \& Aggleton, J. P. (2012). Anterior thalamic nuclei lesions in rats disrupt markers of neural plasticity in distal limbic brain regions. Neuroscience, 224, 81-101.

Dumont, J. R., Wright, N. F., Pearce, J. M., \& Aggleton, J. P. (2014). The impact of anterior thalamic lesions on active and passive spatial learning in stimulus controlled environments: Geometric cues and pattern arrangement. Behavioral Neuroscience, 128(2), 161.

Dupire, A., Kant, P., Mons, N., Marchand, A. R., Coutureau, E., Dalrymple-Alford, J., \& Wolff, M. (2013). A role for the anterior thalamic nuclei in affective cognition: Interactions with environmental conditions. Hippocampus, 23, 392-404.

Duprez, T. P., Serieh, B. A., \& Raftopoulos, C. (2005). Absence of memory dysfunction after bilateral mammillary body and mammillothalamic tract electrode implantation: Preliminary experience in three patients. American Journal of Neuroradiology, 26(1), 195-198.

Frizzati, A., Milczarek, M. M., Sengpiel, F., Thomas, K. L., Dillingham, C.M., \& Vann, S. D. (2016). Comparable reduction in zif268 levels and cytochrome oxidase activity in the retrosplenial cortex following mammillothalamic tract lesions. Neuroscience, 330, 39-49. Gonzalo-Ruiz, A., Alonso, A., Sanz, J. M., \& Llinas, R. R. (1992). Afferent projections to the mammillary complex of the rat, with special reference to those from surrounding hypothalamic regions. Journal of Comparative Neurology, 321(2), 277-299.

Guillery, R. W. (1957). Degeneration in the hypothalamic connexions of the albino rat. Journal of Anatomy, 91(Pt 1), 91.

Jenkins, T. A., Amin, E., Pearce, J. M., Brown, M. W., \& Aggleton, J. P. (2004). Novel spatial arrangements of familiar visual stimuli promote activity in the rat hippocampal formation but not the parahippocampal cortices: a c-fos expression study. Neuroscience, $124(1), 43-52$. 
Jenkins, T. A., Dias, R., Amin, E., \& Aggleton, J. P. (2002a). Changes in Fos expression in the rat brain after unilateral lesions of the anterior thalamic nuclei. European Journal of Neuroscience, 16, 1425-1432.

Jenkins, T. A., Dias, R., Amin, E., Brown, M. W., \& Aggleton, J. P. (2002b). Fos imaging reveals that lesions of the anterior thalamic nuclei produce widespread limbic hypoactivity in rats. Neuroscience, 22(12), 5230-5238.

Kirk, I. J. (1998). Frequency modulation of hippocampal theta by the supramammillary nucleus, and other hypothalamo-hippocampal interactions: mechanisms and functional implications. Neuroscience \& Biobehavioral Reviews, 22(2), 291-302.

Kopelman, M. D. (2015). What does a comparison of the alcoholic Korsakoff syndrome and thalamic infarction tell us about thalamic amnesia? Neuroscience \& Biobehavioral Reviews, $54,46-56$.

Loukavenko, E. A., Ottley, M. C., Moran, J. P., Wolff, M., \& Dalrymple-Alford, J. C. (2007). Towards therapy to relieve memory impairment after anterior thalamic lesions: improved spatial working memory after immediate and delayed postoperative enrichment. European Journal of Neuroscience, 26(11), 3267-3276.

Loukavenko, E. A., Wolff, M., Poirier, G. L., \& Dalrymple-Alford, J. C. (2016). Impaired spatial working memory after anterior thalamic lesions: Recovery with cerebrolysin and enrichment. Brain Structure \& Function, 221(4), 1955-1970.

Nelson, A. J., \& Vann, S. D. (2014). Mammilliothalamic tract lesions disrupt tests of visuospatial memory. Behavioral Neuroscience, 128(4), 494.

Perry, B. A. L., Mercer, S. A., Barnett, S. C., Lee, J., \& Dalrymple-Alford, J. C. (2018). Anterior thalamic lesions have a greater impact than mammillothalamic tract lesions on the extended hippocampal system. Hippocampus, 28(2), 121-135. 
Poirier, G. L., Shires, K. L., Sugden, D., Amin, E., Thomas, K. L., Carter, D. A., \& Aggleton, J. P. (2008). Anterior thalamic lesions produce chronic and profuse transcriptional deregulation in retrosplenial cortex: a model of retrosplenial hypoactivity and covert pathology. Thalamus \& related systems, 4(1), 59-77.

Poirier, G. L., \& Aggleton, J. P. (2009). Post-surgical interval and lesion location within the limbic thalamus determine extent of retrosplenial cortex immediate-early gene hypoactivity. Neuroscience, 160(2), 452-469.

Seki, M., \& Zyo, K. (1984). Anterior thalamic afferents from the mamillary body and the limbic cortex in the rat. Journal of Comparative Neurology, 229(2), 242-256.

Tsanov, M., Chah, E., Vann, S. D., Reilly, R. B., Erichsen, J. T., Aggleton, J. P., \& O'Mara, S. M. (2011). Theta-modulated head direction cells in the rat anterior thalamus. Journal of Neuroscience, 31(26), 9489-9502.

Ulrich, K., Aitken, P. N., Abraham, W. C., Dalrymple-Alford, J. C., McNaughton, N. (2014). Effects of thalamic lesions on repeated relearning of a spatial working memory task. Behavioural Brain Research, 261, 56-59.

Vann, S. D. (2010). Re-evaluating the role of the mammillary bodies in memory. Neuropsychologia, 48(8), 2316-2327.

Vann, S. D. (2011). A role for the head-direction system in geometric learning. Behavioural Brain Research, 224(1), 201-206.

Vann, S. D. (2013). Dismantling the Papez circuit for memory in rats. Elife, 2, e00736.

Vann, S. D., \& Aggleton, J. P. (2003). Evidence of a spatial encoding deficit in rats with lesions of the mammillary bodies or mammillothalamic tract. Journal of Neuroscience, 23, $3506-3514$. 


\begin{abstract}
Vann, S. D., \& Albasser, M. M. (2009). Hippocampal, retrosplenial, and prefrontal hypoactivity in a model of diencephalic amnesia: Evidence towards an interdependent subcortical-cortical memory network. Hippocampus, 19, 1090-1102.
\end{abstract}

Vann, S. D., \& Nelson, A. J. (2018). Anterior thalamic lesions have a greater impact than mammillothalamic tract lesions on the extended hippocampal system: A reply. Hippocampus.

Warburton, E. C., Morgan, A., Baird, A. L., Muir, J. L., \& Aggleton, J. P. (1999). Does

pretraining spare the spatial deficit associated with anterior thalamic damage in rats? Behavioral Neuroscience, 113(5), 956.

Winter, S. S., Wagner, S. J., McMillin, J. L., \& Wallace, D. G. (2011). Mammillothalamic tract lesions disrupt dead reckoning in the rat. European Journal of Neuroscience, 33(2), 371381. 